\title{
2.11. NECESSARY CONDITIONS FOR INTERPOLATION BY ENTIRE FUNCTIONS*
}

Let $p$ be a subharmonic function on $\mathbb{C}$ such that $\log (1+|z|)=O(p(z))$ and let $A_{p}$ denote the algebra of entire functions $f$ such that $|f(z)| \leqslant A \exp (B p(z))$ for some $A, B>0$. Let $\mathrm{V}$ denote a discrete sequence of points $\left\{a_{\mathbf{n}}\right\}$ of $\mathbb{C}$ together with a sequence of positive integers $\left\{p_{n}\right\}$ (the multiplicities of $\left\{a_{n}\right\}$ ). If $f \in A_{p}, f \neq 0$, then $V(f)$ denotes the sequence $\left\{a_{n}\right\}$ of zeros of $f$ and $p$ is the order of zero of $f$ at $a_{n}$.

In this situation, there are three natural problems to study.

I. Zero Set Problem. Given $p$, describe the sets $V(f), f \in A_{p}$

II. Interpolation Problem. If $\left\{a_{n}, p_{n}\right\}=V \subset V(f)$ for some $f, f \in A_{p}$, describe all sequences $\left\{\lambda_{\mathrm{n}, \mathrm{k}}\right\}$ which are of the form

$$
\lambda_{n, k}=\frac{g^{(k)}\left(a_{n}\right)}{k !}, 0 \leqslant k<p_{n}, n=1,2, \ldots \text { for some } g, g \in A_{\rho} \text {. }
$$

III. Universal Interpolation Problem. If $V \subset V(f)$ for some $f, f \in A_{\rho}$, under what conditions on $V$ is it true that for every sequence $\left\{\lambda_{n, k}\right\}$, such that $\left|\lambda_{n, k}\right| \leqslant A \exp \left(B p\left(a_{n}\right)\right)$, there exists $g, g \in A_{p}$, satisfying (1).

In case $p(z)=p(|z|)$ (and satisfies some mild, technical conditions), quite good solutions to problems I-III are known. This work has been carried out by Leont'ev and others (see, e.g., [1] for a survey). However, when $p$ is not a function of $|z|$, the general solutions are not known.

The purpose of this note is to call attention to an interesting special case of III. Consider the case $p(z)=|\operatorname{Im} z|+\log \left(1+|z|^{2}\right)$. Then $A_{p}=\hat{\varepsilon}^{\prime}$, the space of all entire functions of exponential type with polynomial growth on the real axis. The space $\hat{\varepsilon}^{\prime}$ is of special interest because, by the Paley-Wiener-Schwartz theorem, it is the space of Fourier transforms of distributions on $\mathbb{R}$ with compact support. The problems I-III are then dual to some problems about convolution operators on the space $\mathcal{E}=C^{\infty}(\mathbb{R})$ (see, e.g., [1-3]).

Specifically, suppose for some $\varepsilon>0, c>0, f \in \hat{\varepsilon}^{\prime}$, we have

$$
\begin{aligned}
& V=\left\{a_{n}, \rho_{n}\right\} \subset V(f), \text { whe re } \frac{\left|f^{\left(P_{n}\right)}\left(a_{n}\right)\right|}{P_{n} !} \geqslant \varepsilon \exp \left(-\frac{\rho\left(a_{n}\right)}{P_{n}}\right) \\
& \left(p(z)=|\operatorname{Im} z|+\log \left(1+|z|^{2}\right)\right) .
\end{aligned}
$$

Then it is not hard to show that (2) is a sufficient condition that $\mathrm{V}$ has the universal interpolation property III. We wish to pose the converse problem.

Problem. Suppose that $V \subset V(F)$ for some $F, F \in \hat{\varepsilon}^{\prime}$, and that $V$ is a universal interpolating sequence, i.e., III holds. Is it true that (2) must hold for some $f, f \in \hat{\varepsilon}^{\prime}(\mathbb{R})$ ?

In all the cases known to the author where the problem has answer yes, it is also true that the range of the multiplication operator $M_{F}: A_{P} \rightarrow A_{p}$ given by $M_{F}(f)=F f$ is closed. Is the fact that $M_{F}$ has closed range necessary for a "yes" answer? (In the case $A_{p}=\hat{\varepsilon}^{\prime}$, if $M_{F}$ has closed range, then the problem has answer yes, as can be shown by the techniques of [4].) However, the main interest in the problem is to find if (2) must hold with no additional assumptions on $\mathrm{F}$.

*B. A. TAYLOR. Mathematics Department, University of Michigan, Ann Arbor, Michigan 48109. 
1. A. F. Leont'ev, "On properties of sequences of linear aggregates that converge in a region in which the system of functions generating the linear aggregates is not complete," Usp. Mat. Nauk, 11, No. 5, 26-37 (1956).

2. L. Ehrenpreis, Fourier Analysis in Severa1 Complex Variables, Wiley-Interscience, New York $(1970)$.

3. V. P. Palamodov, Linear Differential operators with Constant Coefficients, SpringerVerlag, New York (1970).

4. L. Ehrenpreis and P. Malliavin, "Invertible operators and interpolation in AU spaces," J. Math. Pure Appl., 13, 165-182 (1974).

5. A. I. Borisevich and G. P. Lapin, "On the interpolation of entire functions," Sib. Mat. Zh., 9 , No. 3, 522-529 (1968). 